\title{
Progress on Fabric Electrodes Used in Biological Signal Acquisition
}

\author{
Zhen Liu, Xiaoxia Liu* \\ College of Fashion, Shanghai University of Engineering Science, Shanghai, China \\ Email: "liuxiaoxialucky@126.com
}

Received 27 March 2015; accepted 24 May 2015; published 27 May 2015

Copyright (C) 2015 by authors and Scientific Research Publishing Inc.

This work is licensed under the Creative Commons Attribution International License (CC BY). http://creativecommons.org/licenses/by/4.0/

(c) (i) Open Access

\begin{abstract}
Due to its convenience, wear ability, affinity, continuously monitoring biological signal, etc., fabric electrodes used in biological signal acquisition attracted more and more interest from researchers around the world. Fabric electrode is a kind of intelligent textiles, and its application is very prominent in biological signal acquisition, supercapacitors and ECG (electric signal) monitoring. There are many methods that can be used to prepare fabric electrodes. They are mainly metal plating, conductive polymer coating, magnetron sputtering, and gas phase deposition and impregnation. Besides, they select the appropriate substrate, conductive medium and composite way to get light fabric electrodes which have high conductivity and good conformability. Since the conductive properties of conductive polymers are weaker than those of metal, conductive polymer must undergo protonic acid doping. They can be polythiophene, polypyrrole, polyaniline, etc. However, at present, researchers commit more studies to metal or carbon-based electrodes. For example, a layer of silver film is platted on the fabric by magnetron sputtering to increase its conductivity. In this paper, the development of fabric electrodes was studied from several aspects, namely, the generation of biological signals, the overall design of the wearable monitoring systems, its classification, conductive principles, preparation of fabric electrode and application.
\end{abstract}

\section{Keywords}

Wearable, Biological Signals, Fabric Electrodes, Artifacts

\section{Introduction}

Recently, the quality of life was seriously affected by cardiovascular disease, sleep apnea and other diseases. With the accelerated pace of population aging and the increasing health care costs, more and more attention was

"Corresponding author. 
paid to wearable remote medical monitoring system research. Wearable health monitoring systems typically include signal acquisition, processing systems and information feedback platform and so on. Fabric electrode with a textile structure is an indispensable material in signal acquisition process in which fabric electrode capable of receiving biological signals acts as a signal sensor. It not only can be used to monitor human biological signals constantly, but also will not irritate the skin-causing skin irritation, ulcers, etc. Fabric electrode has many advantages. Its comfortable, breathable, non-irritating properties are greatly favored by many researchers. With the development of health sector oriented toward miniaturization, intelligence and convenience, fabric electrode has become one of the hottest researches among researchers in this area. As a new generation of bio-signal acquisition material meeting the requirements of the development of modern medicine, fabric electrode has characteristics of comfort, intelligence, convenience, and accuracy, different from traditional conductive gel which is prone to cause skin allergies and has some other shortcomings. Future health care industry has two main characteristics. For one, it chooses convenient and accurate medical equipments based on nanomaterial and wearable medical monitoring systems. For another, it combines medical technology, computer technology, and ergonomics to form a both high efficient and humane medical treatment. The function of fabric electrode complies with the two trends mentioned above. If applied to non-contact medical monitoring technology, fabric electrode will bring great market demand. Therefore, fabric electrode has high research value and social and economic benefits.

\section{Generation Principles of Biological Signals \& the Overall Design of the Wearable Monitoring System}

\subsection{Generation Principles of Biological Signals}

Human body is composed of cell membrane which is a kind of semi-permeable membrane. The principle of semipermeable membrane is that only a certain kind of ions can go through it in a certain state [1]. It's known that there are a series of physiological and chemical changes in the body when a man is in motion. When people are in two different states-still and motion, the bio-electric potential in body were respectively resting potential and action potential. At different potentials, the cell membrane has different permeability for sodium ions $\left(\mathrm{Na}^{+}\right)$and potassium $\left(\mathrm{K}^{+}\right)$. That is to say, when the cell is at resting potential, membrane permeability for potassium $\left(\mathrm{K}^{+}\right)$is greater than sodium ions $\left(\mathrm{Na}^{+}\right)$, making potassium ions move to the extracellular, and the membrane potential outside is higher than the membrane potential inside, forming a negative potential inside while positive external. Instead, when the cell is at action potential, membrane permeability for sodium ions $\left(\mathrm{Na}^{+}\right)$is greater than potassium $\left(\mathrm{K}^{+}\right)$, making sodium ions $\left(\mathrm{Na}^{+}\right)$continuously transfer to the intracellular, and the membrane potential outside is lower than the membrane potential inside, forming a negative potential outside while positive internal. To conclude, the so-called EMG (electromyographic signal) and ECG (electric signal) that fabric electrode collected is actually this changing potential difference, as is shown in Figure 1.

\subsection{The Overall Design of the Wearable Monitoring System}

Researchers found that somatic heartbeat, muscle contraction or other action will produce a weak electrical torrent, which are bio-currents. Biological current goes through body fluids and tissues, then spread to the skin surface, thereby creating a potential difference, as is shown in Figure 2. The design thought of wearable monitoring system is that use fabric electrode as electrical sensors to collect biological signals, and pass signals obtained through a series of signal conditioning and micro-processing, then display these processed signals on the human-machine interface (such as personal digital assistant). Fabric electrode, which is a wearable health monitoring system, can touch skin closely, therefore ECG and EMG can be received adequately by fabric electrodes. The signal, after the signal amplified by signal amplifier, go through the high (low) filter to remove interference waves due to the external electric or noise, thus ,acquiring more accurate signals. Zhai et al. [2] use fabric electrodes made of silver-plated fabric to acquire ECG, and then combine it with signal conditioning and micro-processing device, prepared wearable ECG abnormality detection system eventually.

\section{Design and Classification for Fabric Electrode}

Design principles of fabric electrodes are shown in Figure 3. EMG signal received by the conductive fabric wrapped with fill materials, which propagates through wire. Besides, the support pad was used to keep the fabric electrodes and the skin fit together more better. Fabric electrodes are based on textile materials. During the 


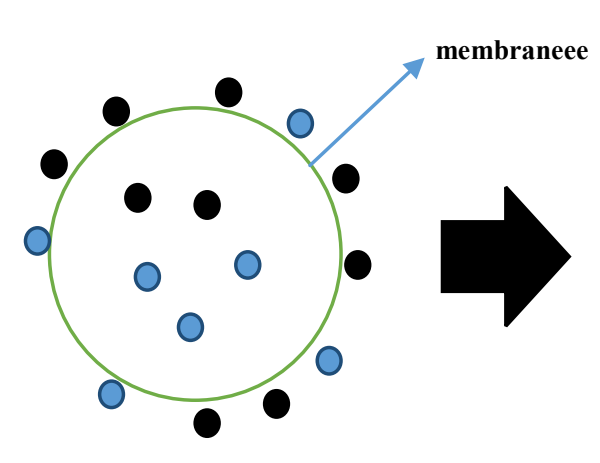

resting potential $(\mathrm{Na}+\mathrm{O}+\mathrm{O})$

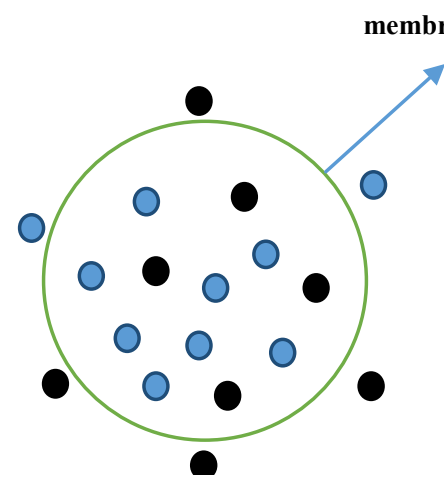

Action potential $(\mathrm{Na}+\mathrm{OK}+\mathrm{O})$

Figure 1. Generation principles of biological signals.

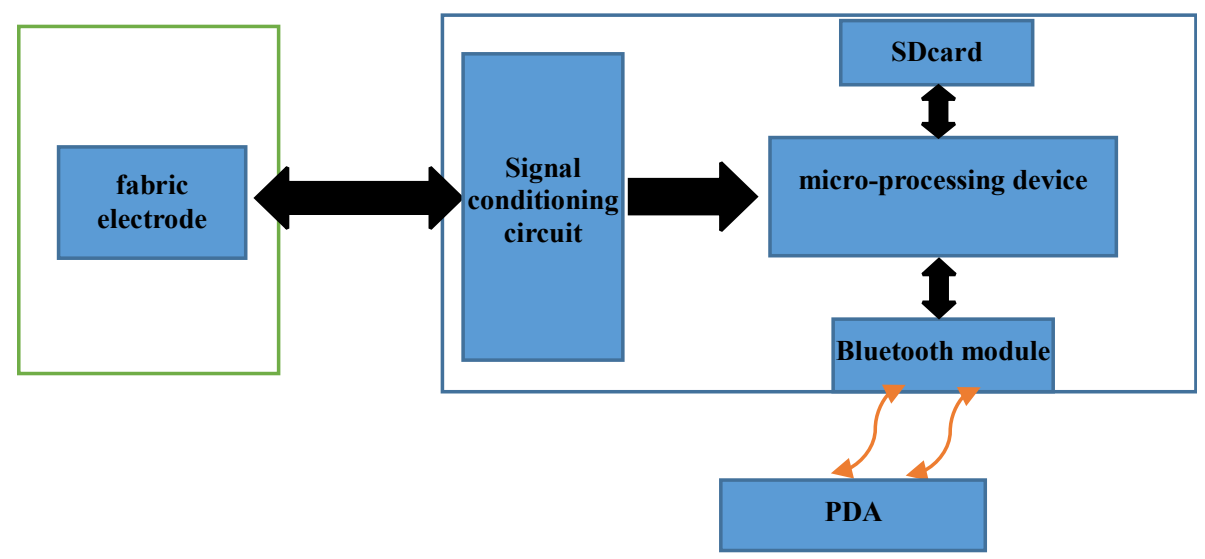

Figure 2. Overall design of the wearable monitoring system.

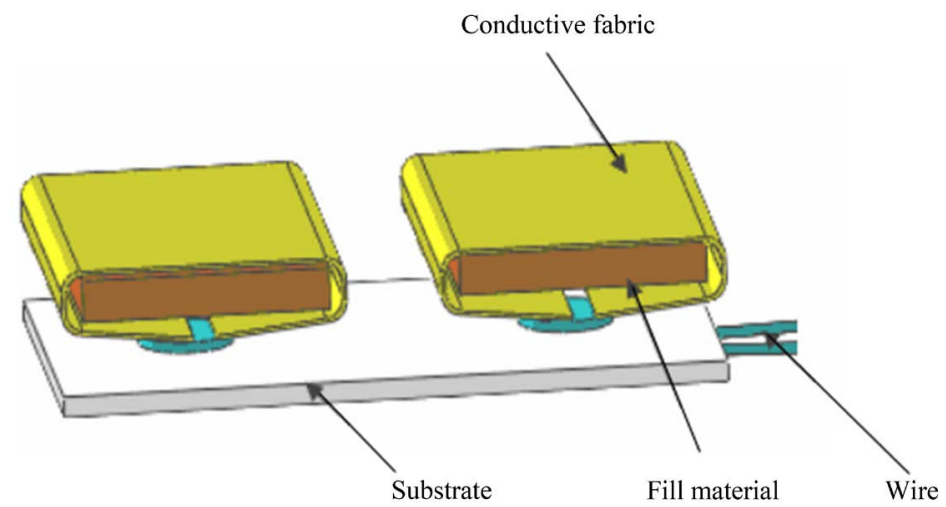

Figure 3. Fabric electrode body design.

post-processing, conductive metal, conductive rubber or conductive ink and other functional materials were attached to the surface of the fabric or yarn by physical or chemical means. As a result, fabric obtains conductive and electrical signals propagate function.

\subsection{Design of Fabric Electrode}

The design of fabric electrode is an integrity layout which includes bio-electrical signal receiving, processing and feedback, etc. It is not only a selection of conductive materials, but also need to consider the size, shape, textile material of fabric electrodes and skin adhesion, etc. 
1) The size of fabric electrode. The size of fabric electrode is a key factor that directly affects the accuracy of biological signals. Experiments show that whenever the size of the fabric electrode is less than $10 \mathrm{~mm}$ or more than $40 \mathrm{~mm}$, it will also reduce the accuracy of the electrodes. According to a series of studies, the larger the size of fabric electrode, the greater the intensity of biological signals received, but if the size is too large, there will be some interfering noise at the contact interface between fabric and skin, seriously affecting the quality of bio-signal acquisition. Therefore, taking the quality and the strength of the signal into account, the diameter of the electrode in the fabric is preferably $10 \mathrm{~mm}$.

2) The shape of the fabric electrodes. The shape of fabric electrode is the main factor affecting potential distribution on the electrode surface. According to studies by related personnel, due to end effects, the uniformity of current distribution on a circular electrode is lower than a rectangular electrode by $30 \%$. It's difficult to describe potential distribution in a limited area. Therefore, Tao et al. dispersed contact surface between electrodes and skin into many small dispersed areas, and assume that each resistance of dispersed contact surface is fixed, similar to two-dimensional Fourier transform which consist of the shape of the electrodes and convolution in signal domain, the impulse response in the spatial region and potential distribution. In this way, Tao et al. [3] respectively found general potential distribution on the surface of rectangular and circular fabric electrodes.

3) The selection of textile materials. When in motion, skin deformation or the interface slip between fabric electrode and skin will produce complex interfering noise, which is called motion artifact. Now studies agree that the movement artifact come from two aspects: for one, the potential change caused by bidirectional disturbance on electrode-skin interface. For another, the interfering noise caused by the squeezing and stretching deformation on the skin. The accuracy of the fabric electrodes, used as a biological signal acquisition component, was seriously affected by motion artifact. Therefore, in recent years, reducing motion artifact is one of the hotspots both at home and abroad in the fabric electrodes area. As the base material of fabric electrodes, textile materials should be dense and thick, otherwise, the body will produce a very serious motion artifact, making SNR (signal to noise ratio) decreasing, further affecting the accuracy of ECG and EMG signals acquisition. The main role of filler material is to make the fabric electrode closely and fully contact with the skin, and reduce SNR and motion artifacts. As a result, it's easy to acquire more real electrical signals [4].

4) Adhesion between fabric electrodes and skin. As a receiving device of body signals, fabric electrode needs to meet the following characteristics. First, because artifacts generated by the relative sliding will seriously affect the SNR, the friction between conductive fabric and the skin should be relatively small. $\mathrm{f}=\mu \times \mathrm{N}$ where, ( $\mathrm{f}-$ the surface friction, $\mu$ - the coefficient of friction surface, $\mathrm{N}$ - a positive pressure). Friction coefficient on skin surface is fixed, and changing the surface roughness of fabric electrodes is also complicated. However, according to $\mathrm{f}=\mu \times \mathrm{N}$, the surface friction increases as $\mathrm{N}$ increases, then the motion artifacts will be reduced. As we know, Wearable fabric deformation can exert a certain pressure on the skin through the support pads and conductive fabric, Therefore, the positive pressure on the skin increases, accordingly, motion artifacts decreasing. Second, it is necessary to minimize artifacts due to the strain caused by the textile substrate, which is a kind of flexible materials. As is shown in Figure 4, conductive cloth should be filled with sponge-like materials to ensure that fabric electrode have a certain three-dimensional shape and a close contact with the skin. In this way, artifacts can largely reduce while SNR increases.

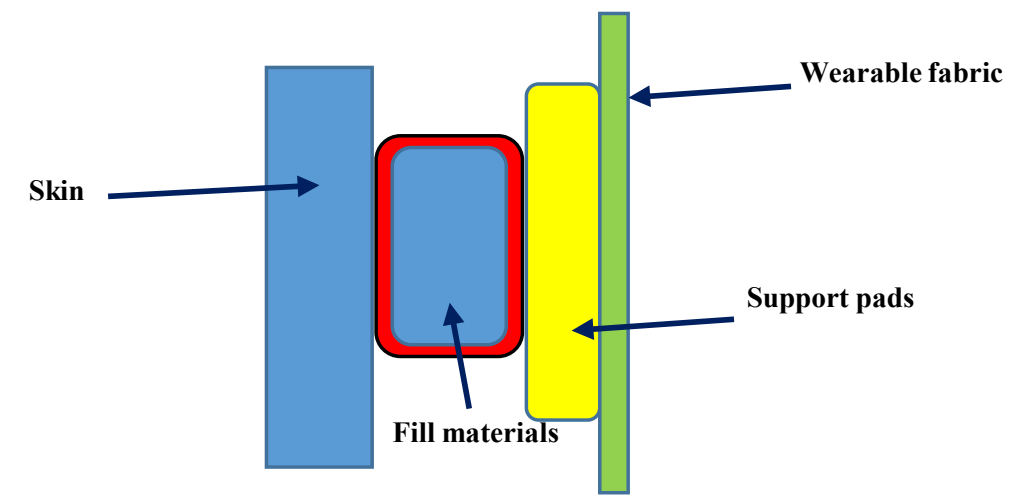

Figure 4. Design of fabric electrode. 


\subsection{Conductive Principle of Electrode Fabric}

Conductive materials of fabric electrode are mainly conductive metal, conductive polymer and conductive ink. Different conductive materials have different conductive mechanism and characteristics.

1) Metal fabric electrodes. Metal is a kind of good conductor, so we can utilize magnetron sputtering or vapor deposition method to uniformly adhere the conductive metal to the fabric, forming a dense conductive metal layer, or in the way of wiring which means disposing conductive metal inside the fabric or yarn surface for transferring electrical signals.

Nano-silver plated fabric material is produced in the way of vacuum coating by Fiber Co., Ltd. Jinan Li Site. Specifically, cross a layer of silver film on polyester or nylon with the method of flexible multi-target magnetron sputtering coating technology, and then silver it with plating treatment again. Fabric electrode prepared by this process has the characteristics of a uniform coating, strong adhesion, washable and so on. In addition, it not only retains the original silk weaving resistance, but also has a lot of advantages in terms of performance of apparel, such as anti-bacterial deodorant, strong interfacial bonding strength, low surface resistance.

2) Polymer fabric electrode. Fu et al. [5] from Donghua University use polyaniline as an intermediate flexible conductive layer to connect metal and polyester fabric. Under ultrasound conditions, with a chemical coating method, they prepared polyaniline polymer/Cu composite conductive fabric, which use polyester as base materials. Conducting polymer is a polymer of conjugated double bonds, which is generated by alternating single and double bonds. Polymer conjugate effect results in mutual handover, overlapping coverage between the adjacent electron clouds so that there will appear a continuous electron cloud in the main chain direction, and then the unpaired electrons can move along the long-chain. Polythiophene, polypyrrole and polyaniline are called the three conductive polymers, wherein polythiophene has the strongest conductive capability while polyaniline has the lowest price. Taking polyaniline as an example, the structure of polyaniline is divided into benzene type and quinone type. There only exists benzene rings in benzene structure, and the large $\pi$ bond electrons are confined to a benzene ring, so the electron cannot move across the polymer chain. Ring structure of quinoid structure of polyaniline is composed of a benzene ring and a quinoid ring together alternately. Large bonds on the benzene ring and the $\pi$ bonds on the quinone ring form conjugated structures, which have overlapping electron cloud, so that electrons are able to move along the polymer chain. Polymer can be called a conductive polymer, only when it meets two conditions as follows: for one, there should be a certain number of current-carrying electrons; for another, there exists overlapping electron clouds (a conductive channel) inside the polymer. Only with the above two conditions, can the polymer be used as the polymer conductive layer of fabric electrode. If we add some protonic acid into polyaniline, benzene structure will be transferred to the quinoid structure. This results in a non-uniform charge amount in the nitrogen $(\mathrm{N})$ atom. Thus a sufficient amount of a carrier and conductive paths in the intramolecular chain is formed. A fabric electrode can be prepared by attaching conductive polymer to its surface through spraying or coating technology [6]-[9].

3) Conductive ink fabric electrodes. As an electronic key material, conductive ink technology are extensively applied in the fabric electrodes. Conductive inks are prepared when conductive polymer or some other conductive functional units are coupled together by a phenolic resin, etc. Fabric electrodes are obtained when conductive ink was attached onto the fabric. The conductive mechanism of conductive ink fabric electrodes can be explained from three aspects, and they are the tunneling effect, field emission effect, Percolation [10]-[15]. a) Tunneling effect. Tunnel theory holds that when the thickness of isolation layer between conductive particles is less than $10 \mathrm{~nm}$, even if there is no direct contact between conductive particles, an electron tunnel effect can still form to produce electron flow. b) Field emission effect. According to the field emission effect, under the effect of the field, as long as the adjacent ion with potential difference is close enough, electrons can be dramatically insulating interfacial layer, then jump to the adjacent conductive ion and generates a current, and non-contactconductive is achieved. c) Percolation. Based on percolation, only when the amount of conductive particle in the resin is large enough and the distance between conductive particles is less than $1 \mathrm{~nm}$ or contact with each other, are the conductive particles capable of electric conduction. These three theories demonstrate that choose conductive ink as the conductive material of textile electrode is feasible. In terms of material, conductive ink can be divided into inorganic conductive ink and organic conductive ink. Inorganic conductive inks mainly choose metal powder ( $\mathrm{Au}, \mathrm{Ag}, \mathrm{Cu}, \mathrm{AL}, \mathrm{Ni}$, etc.) or a conductive carbon black or carbon fibers as its conductive functional material. Inorganic conductive ink has many advantages such as stability, high efficiency. In recent years, the inorganic conductive material becomes the main research field of the conductive ink fabric electrode. Under 
Table 1. Comparison between organic conductive ink and inorganic conductive ink.

\begin{tabular}{|c|c|c|c|}
\hline Classification & Functional unit & & Comprehensive performance \\
\hline \multirow{6}{*}{ Inorganic conductive ink } & $\mathrm{Au}$ & $\begin{array}{l}(1) \\
(2)\end{array}$ & $\begin{array}{l}\text { Good performance } \\
\text { High price }\end{array}$ \\
\hline & $\mathrm{Ag}$ & $\begin{array}{l}(1) \\
(2)\end{array}$ & $\begin{array}{l}\text { Conductivity behind } \mathrm{Au} \\
\text { Sensitive to temperature }\end{array}$ \\
\hline & $\mathrm{Cu}$ & $\begin{array}{l}(1) \\
(2) \\
(3)\end{array}$ & $\begin{array}{l}\text { Wide application } \\
\text { Easy oxidation } \\
\text { High cost performance }\end{array}$ \\
\hline & $\mathrm{Al}, \mathrm{Ni}$ & $\begin{array}{l}(1) \\
(2) \\
(3)\end{array}$ & $\begin{array}{l}\text { Easily oxidized } \\
\text { Low price, unstable; } \\
\text { Ordinary conductivity }\end{array}$ \\
\hline & Metal nanopowders & $\begin{array}{l}(1) \\
(2)\end{array}$ & $\begin{array}{l}\text { Good conductivity } \\
\text { Conductivity varies with different powder }\end{array}$ \\
\hline & Conductive carbon black, carbon fibers & $\begin{array}{l}(1) \\
(2) \\
(3)\end{array}$ & $\begin{array}{l}\text { Poor conductivity } \\
\text { Poor moisture resistance } \\
\text { Low price difference }\end{array}$ \\
\hline Organic conductive ink & Doped conducting polymer & $\begin{array}{l}(1) \\
(2)\end{array}$ & $\begin{array}{l}\text { Low temperature curing } \\
\text { Easy to use }\end{array}$ \\
\hline
\end{tabular}

the effect of doped conjugated polymer, conductive properties of organic conductive ink are enhanced. At the same time, at the macro level, it also retains the characteristics of polymer materials such as mechanical properties and processability, etc. Therefore, organic conductive ink has become another important research direction of ink conductive fabric electrodes. The comparison between organic conductive ink and inorganic conductive ink is shown in Table 1.

As functional conductive materials, Au has excellent comprehensive properties, but its price is high. The conductivity of $\mathrm{Ag}$ is only second to that of $\mathrm{Au}$, and Ag has good antibacterial property. Although Ag is sensitive to temperature, this does not affect the normal usage of fabric electrode. $\mathrm{Cu}$ has a high cost performance and wide application, but easy to be oxidized. The conductivity of $\mathrm{Al}$ and $\mathrm{Ni}$ is ordinary, and they are easily oxidized, but the price is cheaper. Therefore, $\mathrm{Al}$ and $\mathrm{Ni}$ can be chosen to prepared disposable fabric electrodes. When it comes to conductive carbon black and carbon fiber, due to its poor conductive property and poor moisture resistance, whether it is suitable to be used as functional conductive materials still needs further research. The conductive ratio of intrinsic conducting polymer is low, and its conductive ratio generally maintained at $10^{-10}$ level of S/cm. After doping with chemical or electrochemical method, conductive rate can be greatly improved to $10^{-9}$ $\mathrm{S} / \mathrm{cm}$ to $10^{5} \mathrm{~S} / \mathrm{cm}$. But considering its high manufacturing cost, complicated technology and difficult to control the doping level and unstable nature. In addition, compared to the other conductive materials, its resistivity is high, so further research is needed.

\subsection{Preparation of Fabric Electrodes}

Fabric electrode is different from traditional conductive gel, which is based on textile materials, and has unique advantages of fabrics such as breathability, flexibility, foldability. Fabric electrodes is different form conductive gel, which is easy to cause skin irritation or symptoms such as swelling when touching the skin for a long time, and greatly affect the use of the wearer [16] [17]. As for needle electrodes, in order to be able to accurately capture the electromyographic signal, needle electrodes often need to be inserted into human's body skin to collect potential of local motion unit activities. With this method, we can get more accurate electromyographic signals, but it will inevitably cause mechanical damage to the human's body. Fabric electrodes are no harm to human body. However, unlike needle electrodes, fabric electrode cannot acquire action potential collected from local motion unit. With the improvement of SNR and the artifact technology, Fabric electrodes still have the very high research value. Comparison of different electrodes is shown in Table 2.

\subsection{The Choice of Conductive Functional Materials}

The choice of electrode conductive fabric functional materials mainly depends on the fabric electrode conduc- 
Table 2. Comparison of different electrodes.

\begin{tabular}{ccccc|}
\hline & Fabric electrode & Traditional metal electrode & Patch electrode & Needle electrode \\
\hline Conductive medium & Inert metal and chlorine & Inert metal & Patch electrode & Metal \\
Time-using & Long & Short & Short & Short \\
Skin allergy & No & Yes & Yes & No \\
Skin damaging & No & No & No & Yes \\
Comfort level & High & Normal & High & Feel painful \\
Operability & High & High & & Low
\end{tabular}

tive mechanism. As for preparation for fabric electrode medical care system, we cannot require electrode materials thin and soft exceedingly. Because the electromyographic signal (EMG) and electric signal (ECG) are very weak. The relative friction and the fabric sliding due to human body mechanical movement will largely affect the accuracy of fabric electrode acquisition signal. Therefore, in order to improve the signal-to-noise ratio and reduce the motion artifact, we need to choose dense and thick material as the base of fabric electrodes. With $76 \%$ of medical grade of the silver-plated nylon and $24 \%$ of spandex fabric, Ding et al. [18] have made some fabric electrodes, which is density, conductivity of $0.6 \Omega / \mathrm{cm}^{2}$ of non-contact sleeping monitoring system. Conductive functional elements used in acquisition of bioelectric signals are respectively electrically conductive thread, conductive rubber and conductive ink, and they are conventional conductive substrate of fabric electrodes. Their advantages and disadvantages are as follows.

1) Conductive thread

Electrically conductive thread is directly prepared by metal. When choose conductive thread as functional conductive material of fabric electrodes, fabric electrode and biological electrical signal analysis instrument can be directly connected by conductive thread. Inert metal is generally selected as conductive wire metal, because its chemical properties are quite stable, and it is not easy to cause allergic problem such as redness and swelling. But because the conductive yarn is made of hard metal, when contacting with fabric electrodes, the skin will be easy to hurt by friction, greatly affecting the mood of users. In addition, unlike fabrics, metal can't fit with the body surface; on the contrary, it will produce great deformation. Therefore, it is easy to form artifact due to human body's mechanical motion, and affects the quality of the biological electrical signal acquired, making the signal-to-noise ratio very low. The conductive thread is attached with a layer of dense conductive metal film on its surface with the method of vapor deposition and magnetron sputtering. Coated conductive polymer thread making process is as follows. In order to reduce the resistivity, polythiophene, polypyrrole and polyaniline and other polymer with conjugated groups should be doped, and then coated a layer of compact conductive polymer film on the surface of yarn.

2) Conductive rubber

In terms of conductive rubber, first, fill conductive carbon, metal or conductive composite material into the silicone rubber [19], and then obtain fabric electrodes by method of fast styling or printing. Rubber is soft and no stimulation to the skin. When the human body is doing mechanical movement, it deforms along with the skin deformation. Therefore it can effectively reduce the signal error due to motion artifact.

3) Conductive ink

The main composition of conductive ink is silver powder and petroleum jelly. Fabric electrode, made of conductive ink, have a very small surface friction coefficient, thus it won't cause damage to skin due to mechanical friction. Yue and Ding [20] plate gold film on fabrics by magnetron sputtering, then deal with acetonitrile solution, which containing toluene sulfonic acid and polypyrrole monomer, by chemical polymerization method polymerization methods. Thus, they obtained the polypyrrole composite conductive fabric, one kind of fabric electrodes. Xu et al. [21] firstly coat a layer of metal nickel on fabric surface, and then deposit the catalytically active conductive polymer on the nickel. Thus, they obtain a dye-sensitized solar fabric electrode.

\section{Applications of Fabric Electrodes}

\subsection{Applications of Fabric Electrodes in ECG Monitoring}

With the development of medical toward miniaturization, convenience, efficiency, in the health field, medical 
equipment that can continuously monitor ECG signal and the physiological parameters of patients at high risk are in great demand. As long as researchers consider fabric electrodes can be used to monitor chronic diseases, wearable health monitoring systems become a hotspot of modern medical researches. Fabric electrodes choose textile material as substrate, and can be worn. It not only decrease the irritation to the skin and the SNR which traditional conductive gel, conductive membrane electrodes cause, but also not affect the patient's normal activities [22]-[24].

Zhai et al. sewn silver-plated fabric electrodes inside the clothes. With support pad and filler, they make it a good contact with human skin so that fabric electrodes can collect ECG signal. The signal collected pass through the data analysis device first, and then feedback to the user through the PDA. Thus, wearable fabric electrode ECG detecting system is achieved. Wearable fabric electrodes demonstrate the advantages of fabric electrodes, which fully show that fabric electrodes ECG monitoring can be well used in the medical field.

\subsection{Application of Fabric Electrodes in Myoelectric Prosthesis}

Electrical signal collecting devices in traditional myoelectric prostheses are conductive gel electrodes. However, the conductive gel has a poor compatibility to human skin, and cannot be well applied in clinical myoelectric prostheses [25]-[26]. As the original model of the EMG signal acquisition, fabric electrodes retains many unique advantages of textile, namely, breathable and comfortable, washable, folding and affinity for human skin and other advantages. It meets the requirements of clinical medicine, and can replace the traditional disposable electrode. Thus, it will become the future development trend of myoelectric prostheses information acquisition device. Currently, in order to control prosthetics freely, researchers have attempted to collect nerve signals from the cerebral cortex or scalp surface or by implantation of an electrode. Tao et al. made conductive silver paste and fabric into an electrode with a center distance of $20 \mathrm{~mm}$, a pitch of $10 \mathrm{~mm}$, an area of $200 \mathrm{~mm}$. As is shown in Figure 5, fabric electrodes are fixed by elastic belt, and used as a collecting element of EMG signals during mechanical movement.

The researchers asked 11 participants to fix fabric electrodes shown in Figure 5 on their right forearm in the way shown in Figure 6. Each participant's hand should in turn open, make a fist, hold keys, grip, fine pinch grip, grip the cup, and their wrist do stretching, bending, pronation external rotation in turn, finally keeping in a relaxed state.

With the virtual human-machine interface system, participants can control the action of virtual human in Figure 7. Virtual human-machine interface system records the completion of action, time and accuracy to judge its complete state.

\subsection{Application of Fabric Electrodes in Supercapacitors}

The supercapacitor is also called double layer capacitor [27] [28]. It uses the electric double layer, which is formed by the positive and negative ion separation in the porous electrode and electrolyte interface, to store energy. Not only it has the excellent performance of pulse discharge and a large capacity of energy storage, which physical capacitors do not have, but also its power density and service life is higher than the secondary battery. All sensors, drives and other electronic components on smart textiles need providing power, so there should be a light convenient energy storage and conversion device, which has a certain tensile properties. Therefore, the solution is to put this type of power into the fabric.

At present, electrode materials used in the supercapacitor are mostly active carbon, active carbon fiber and carbon nanotubes, etc. At home and abroad, researchers focus their attention of electrode materials on improving specific capacitance and aperture regulation. The resistance of porous carbon electrode materials is mostly large, therefore studies on porous carbon electrode materials is less. Surface metallization is an effective method of improving conductivity and reducing the resistance. Specifically, nickel plate on the surface of activated carbon fabric so that the conductivity improves, therefore the resistance of electrode reduces and power density improves.

According to comparison of the existing research of polypyrrole coated fabric, Binbin Yue analyzed changes of polypyrrole coated fabric in electrical properties and electrochemical properties under different stretched state, and choose it as electrode materials of stretchable supercapacitors, thus he find an effective approach for the preparation of the wearable power device. China Taiwan's comprehensive research Institute of textile industry has developed a foldable fabric supercapacitor, which has won the award in Orlando. It is a kind of lightweight 


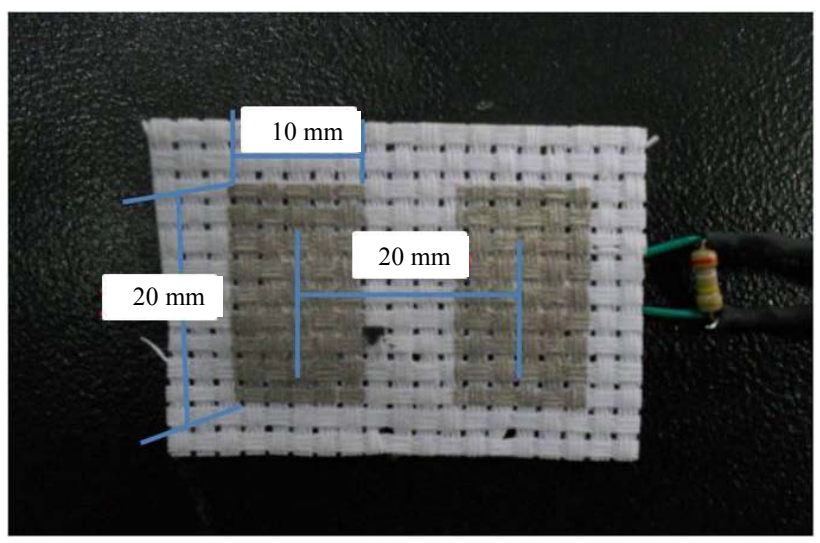

Figure 5. Screen printing method for electrode fabric preparation.

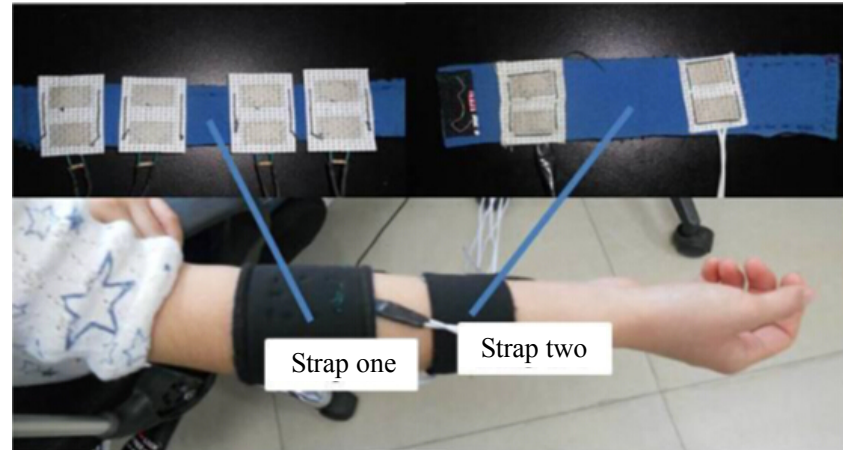

Figure 6. Wearing fabric electrodes to collect biological signals.

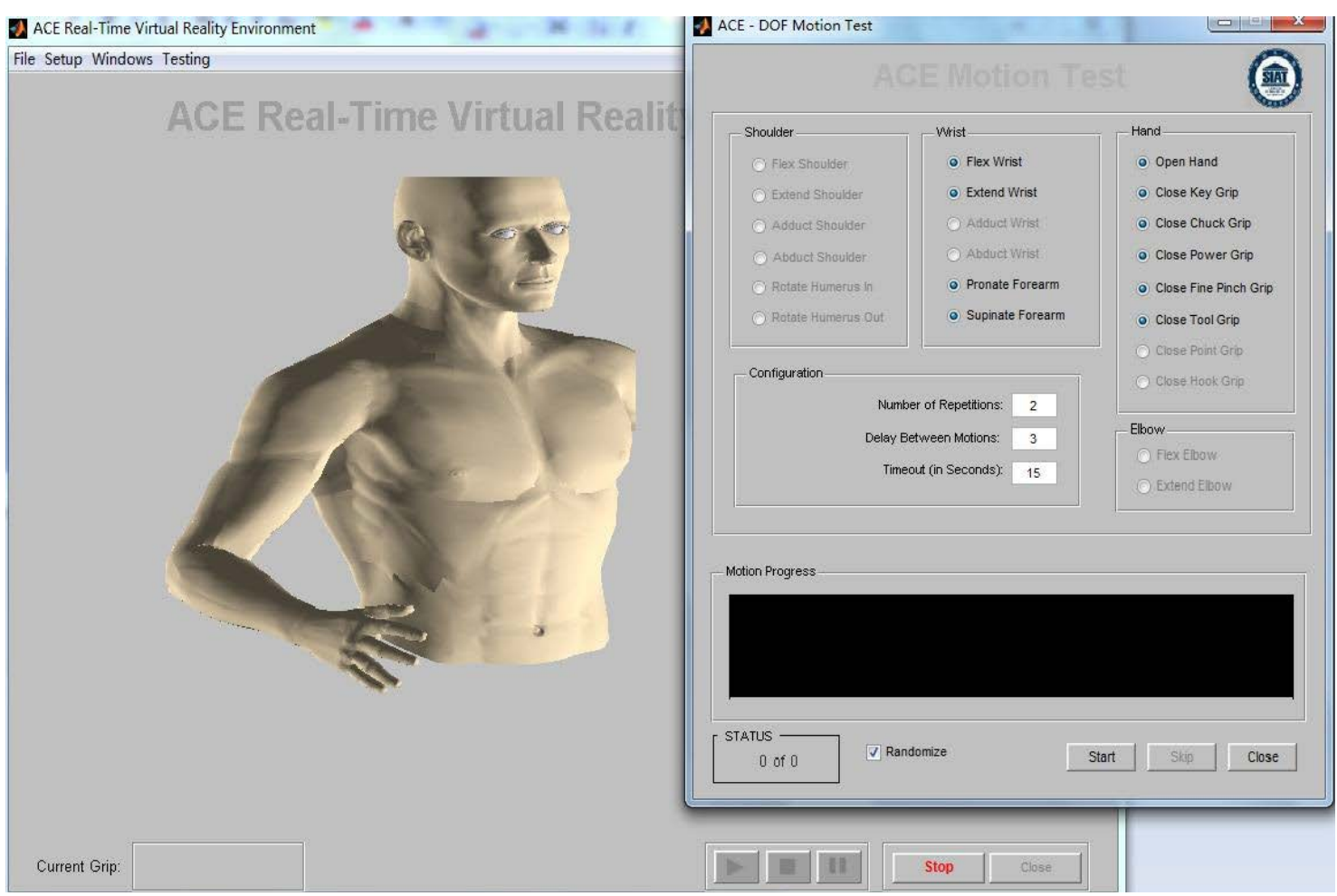

Figure 7. Virtual machine interface systems. 
supercapacitor, which can be completely folded. Fabric electrode, a kind of smart textile, is one of projects initiated by the five-year plan. At home and abroad, it has been about twenty years since researchers studied fabric electrodes which were applied to biological signal acquisition.

Fabric electrode involves many fields such as human body engineering, electronics, biology, textile science, etc. In the domestic and overseas, there is no breakthrough about characterization of the surface potential, motion artifact reduction SNR improvement and so on. Therefore, the research content is very broad, and the research direction is very clear.

\section{Significance of Research on the Textile Electrodes}

Along with the improvement of living standards, people are increasingly seeking light portable concepts. As new electronic products, textile electrodes played a significant role in the patients' ECG monitoring, control of myoelectric prostheses and supercapacitors, etc. New fabric electrode, an electrical signal receiving device, must be subject to the concern of the electronics industry in the era of pursuing miniaturization, intelligent and highefficiency electronic. Fabric electrode can be easily folded, and has good skin-friendly and breathable features. Compared with the conductive gel, long-term use will not cause skin irritation. Due to its rich research content, fabric electrodes bear broad opportunities and prospects, as functional products transform to intelligent products.

\section{References}

[1] Pereira, T., Carvalho, H., et al. (2013) Wearable Biopotential Measurement Using theTIADS1198 Analog Front-End and Textile Electrodes. IEEE International Symposium on Medical Measurements and Applications Proceedings (MeMeA), Gatineau, 4-5 May 2013, 325-330.

[2] Zhai, H., Wang, C., et al. (2012) ECG Signal Monitoring System Based on Textile Electrodes. Journal of Jilin University (Information Science Edition), 30, 185-191.

[3] Tao, D. (2011) Study of Textile Electrodes and Signal Processing Technology in Myoelectric Prostheses Control. Harbin Institute of Technology, Harbin.

[4] Macias, R., Fernandez, M. and Bragos, R. (2013) Textile Electrode Characterization: Dependencies in the Skin-Clothing-Electrode Interface. Journal of Physics: Conference Series, 434, 1-4. http://dx.doi.org/10.1088/1742-6596/434/1/012024

[5] Fu, X., Zhao, Y. and Cai, Z. (2010) Preparation of Polyaniline/Metal Composite Conductive Fabric by Electroless Plating. Dieing Polymerica Sinia, 36, 1-4.

[6] Jing, X., Wang, L., et al. (2005) Synthesis, Structure, Properties and Applications of Conducting Polyaniline. Acta Polymerica Sinia, 5, 655-663.

[7] Liang, G., Zhu, L., Xu, J., et al. (2013) Investigations of Poly(pyrrole)-Coated Cotton Fabrics Prepared in Blends of Anionic and Cationic Surfactants as Flexible Electrode. Electrochimica Acta, 103, 9-14. http://dx.doi.org/10.1016/j.electacta.2013.04.065

[8] Shi, H. (2009) Polyaniline and Derivatives/Preparation Inorganic Composite Materials and Studies on Its Conductivity. Northwest Normal University, Lanzhou.

[9] Zhang, P., He, Y., et al. (2014) Research Progress in Improving the Electrical Conductivity of Polymer Materials. Packaging Journal, 4, 31-38.

[10] Chen, L. and Yu, J. (2008) Study on Preparation and Properties of Modified Epoxy Resin-Based Conductive Ink for Flexible Printed Circuits. Chemistry Bioengineering, 25, 25-27.

[11] Yang, X. and He, W. (2011) Study on Preparation and Properties of the Conductive Ink Composite Materials. Science Technology and Engineer, 11, 3703-3708.

[12] Yang, K., Freeman, C., Torah, R., Beeby, S. and Tudor, J. (2014) Screen Printed Fabric Electrode Array for Wearable Functional Electrical Stimulation. Sensors and Actuators A: Physical, 213, 108-115. http://dx.doi.org/10.1016/j.sna.2014.03.025

[13] Li, L.H., Mo, L.X., Ran, J. and Xin, Z.Q. (2014) Conductive Ink and Its Application Technology Progress. Imaging Science and Photochemistry, 32, 393-401.

[14] Ma, X.F. and Li, F. (2010) Conductive Ink Technology and Research Direction. China Printing and Packing Study, 2, 9-14.

[15] He, W., Yang, Y., Wang, S.X., He, B. and Hu, K. (2009) Preparation Technology and Application Progress of Conductive Inks. Materials Review, 23, 30-33. 
[16] Jang, S., Cho, J., Jeong, K. and Cho, G. (2007) Exploring Possibilities of ECG Electrodes for Bio-Monitoring Smart Wear with Cu Sputtered Fabrics. Lecture Notes in Computer Science, 4551, 1130-1137. http://dx.doi.org/10.1007/978-3-540-73107-8_124

[17] Wang, R.X., Tao, X.M., Wang, Y., Wang, G.F. and Shang, S.M. (2010) Microstructures and Electrical Conductance of Silver Nanocrystalline Thin Films on Flexible Polymer Substrates. Surface and Coatings Technology, 204, 1206-1210. http://dx.doi.org/10.1016/j.surfcoat.2009.10.030

[18] Ding, X., Jin, L., Liu, N. and Chen, Y. (2012) Design of Non-Contact Portable Sleep ECG Monitoring System Based on Fabric Electrodes. Beijing Biomedical Engineering, 31, 293-297.

[19] Lei, H.J., Gong, W.F., Wu, J., Wang, M.L. and Zhen, Y.S. (2005) Effect of Metal Filler on Properties of Conductive Silicone Rubber. China Rubber Industry, 52, 667-669.

[20] Yue, B., Wang, C., Ding, X. and Wallace, G.G. (2013) Electrochemically Synthesized Stretchable Polypyrrole/Fabric Electrodes for Supercapacitor. Electrochimica Acta, 113, 17-22. http://dx.doi.org/10.1016/j.electacta.2013.09.024

[21] Xu, J., Li, M., et al. (2013) One Kind of Dye-Sensitized Solar Cell Fabric Electrode and Its Preparation Method. Chinese Patent No. 103258650.

[22] Xi, X.G., Zuo, J., Zhang, Q.Z. and Luo, Z.Z. (2014) A Study of Multi-Channels EMG De-Noising and Aliasing Removal. Chinese Journal of Sensors and Actuators, 2014, 293-298.

[23] Zhang, Y. (2014) Study on Wearable Ambulatory Electrocardiogram Monitoring System and Electrocardiosignal Processing Method. Master's Thesis, University of Electronic Science and Technology of China, Chengdu.

[24] Zhu, D. (2008) Non-Contact ECG Detection System. Master's Thesis, Southwest Jiaotong University, Chengdu.

[25] Bu, F., Li, C.-J., Chen, J.-J., Li, H. and Guo, W.-H. (2014) Design of Electromyography Prosthesis Controller Based on ARM. Journal of Shanghai University (Natural Science Edition), 20, 442-449.

[26] Zhang, Z. (2010) Research on Acquisition of Electromyography for Prosthesis Control. Master's Thesis, Harbin Institute of Technology, Harbin.

[27] Lee, S., Lee, Y., Park, J. and Choi, D. (2014) Stitchable Organic Photovoltaic Cells with Textile Electrodes. Nano Energy, 9, 88-93. http://dx.doi.org/10.1016/j.nanoen.2014.06.017

[28] Babu, K.F., Siva Subramanian, S.P. and Kulandainathan, M.A. (2013) Functionalisation of Fabrics with Conducting Polymer for Tuning Capacitance and Fabrication of Supercapacitor. Carbohydrate Polymers, 94, 487-495. http://dx.doi.org/10.1016/j.carbpol.2013.01.021 